\title{
Puzzling materials
}

$K \cup R T D Y R$ I F P D R G O I B G J X S N I D S E U P

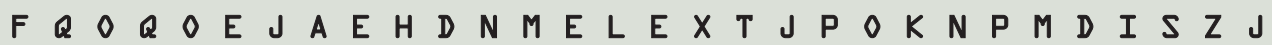

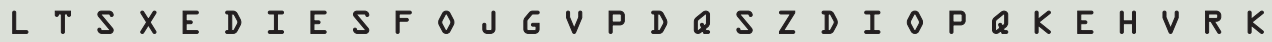
C E T B WC P V O E A Y Q N P C N S M Z T I D X L A K P P V $R L S Z C A Q M C W B Z Y U E L G P A S A F S E C I B C O Q I$ $P B N H K X C F T N N U H Q A X Q Q V U U Z V C C O C Y I L K N K$ $X$ A L L N H Q F K S U S T A I N A B I L I T Y J J O Y $F C Q M Y S E S K Z B E E O Q O O T S Y G R E N E M R C L L O$ G S P K G M R F Y C P U M T N K W U B O E A L E X O Q R G B $X P K X S K O O O B L I Z R K N A N O M A T E R I A L S Q W A$ $P U K J U T J H T H E R M O D Y N A M I C S Y Y S G V P T N I$ $P X W D U V E M H C S M T R R Y G S L T A X C R X E L Y Y M$ $Q L F S I S T J U J U Z G C M N P Q R K R$ I A Z R F R V I S Y C L T S P B X L U M D S S E S Y O L L A P C O W C P O M T $P A V N$ Y F C V Z E W Q N T E L N O A T H C V W E H D K Y A $Z X M U B C Z B U K Y A I O V I E G L E C S O N H M V S B Z$

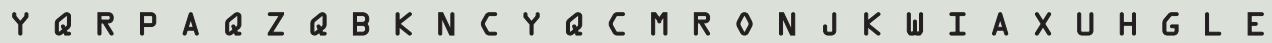

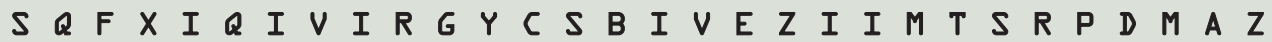
$Z$ Z $G N Z Z O C Y$ T $V$ A C A E R B I O M A T E R I A L S D B T B V $T R K T$ T I G I J L Z V B D X X T K N $N$ E H T I E Z Q M Y $Q R \times I H F X S F M J T W O P H S T S A$ A P E Q N X E Y P W $V L B E L J I H I H E I H Z G R V P C A R B O N G F V W X F$ $G \vee M E K J B H X O X P Z P M G R C G J F E I N I Y Y S X P O$ $R U S Y O K H F W Z C J B E W N K R V G O M W J B G W M M A$ $C L R L E L G I U Y A L O N O C Z S H A S Q N U$ U I A Q N W C C $M B L G R V H O K Y H Z I U G R S S I F C R R V R B C E X P$ $Z M Q Y M Q K F$ I K L C U Z B J W M T C P T T $D V L L U T X L$ E S B I Y I N F O Z L X K X O E B Z M H K B Z X H I S F B D $B C V U H V P Y Y X X T W L Z X F B L Y B \quad Y U E Z Q Q M X P R \quad D$ $F F I R Z V B \quad D B H O H A T Q T N L A M U N N E N I J C P V$
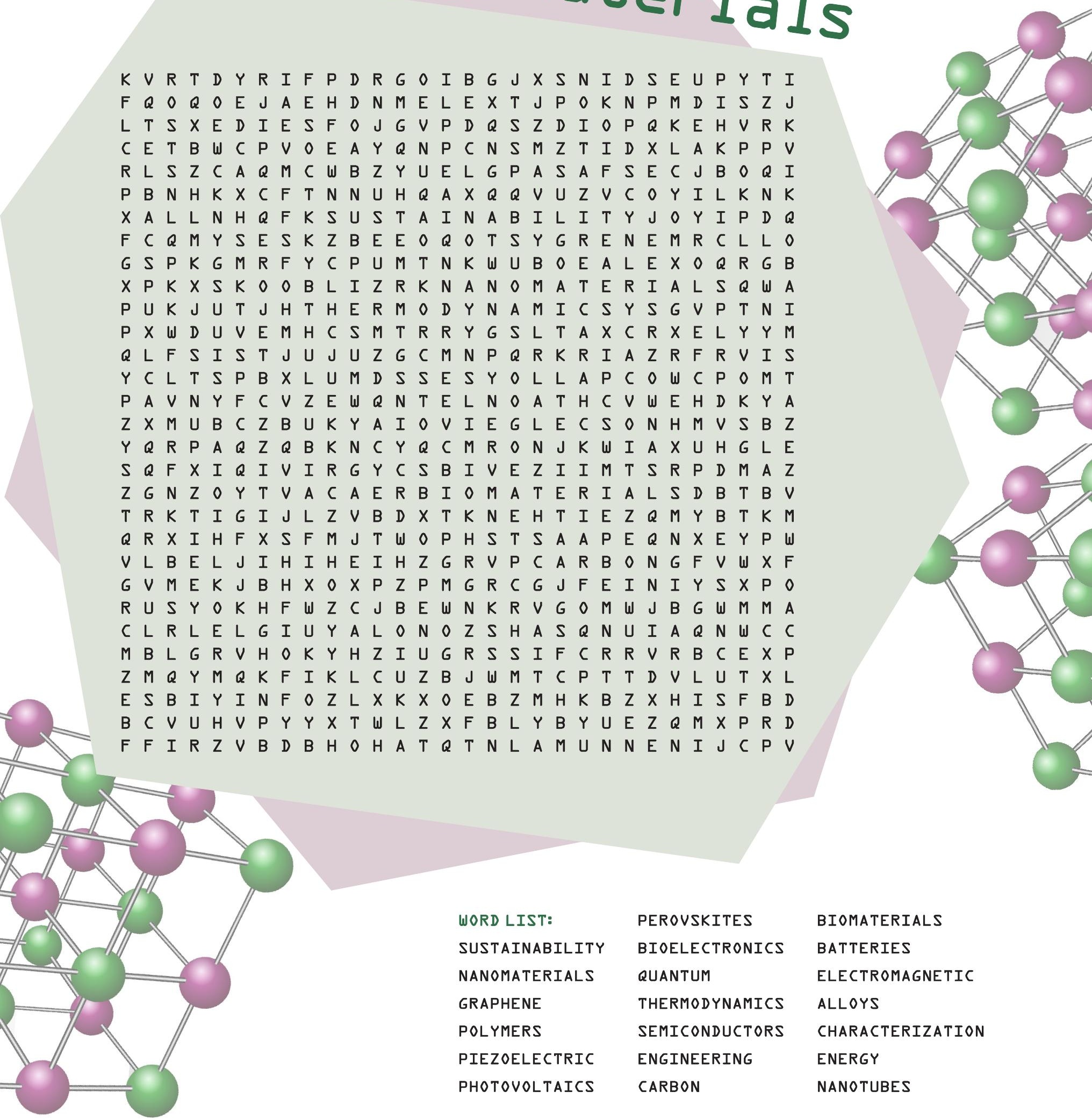

WORD LIST:

SUSTAINABILITY NANOMATERIALS

GRAPHENE

POLYMERS

PIEZOELECTRIC

PHOTOVOLTAICS
PEROVSKITES

BIOELECTRONICS

QUANTUM

THERMODYNAMICS SEMICONDUCTORS ENGINEERING CARBON
BIOMATERIALS

BATTERIES

ELECTROMAGNETIC

ALLOYS

CHARACTERIZATION

ENERGY

NANOTUBES 